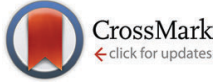

Cite this: Phys. Chem. Chem. Phys., 2015, 17, 27653

Received 22nd August 2015 Accepted 16th September 2015

DOI: $10.1039 / c 5 c p 05005 g$

www.rsc.org/pccp

\section{Synthesis of magnetic mesoporous titania colloidal crystals through evaporation induced self-assembly in emulsion as effective and recyclable photocatalysts $\dagger$}

\author{
Jeffrey E. Chen, $\ddagger^{\mathrm{a}}$ Hong-Yuan Lian, $\dagger^{\mathrm{a}}$ Saikat Dutta, ${ }^{a}$ Saad M. Alshehri, ${ }^{\mathrm{b}}$ \\ Yusuke Yamauchi, ${ }^{\text {bc }}$ Mai Thanh Nguyen, ${ }^{d}$ Tetsu Yonezawa*d and Kevin C.-W. Wu*a
}

\begin{abstract}
This study illustrates the directed self-assembly of mesoporous $\mathrm{TiO}_{2}$ with magnetic properties due to its colloidal crystal structure with $\mathrm{Fe}_{3} \mathrm{O}_{4}$. The $\mathrm{Fe}_{3} \mathrm{O}_{4}$ nanoparticles were synthesized using co-precipitation techniques to a size of $28.2 \mathrm{~nm}$ and a magnetic saturation of $66.9 \mathrm{emu} \mathrm{g}^{-1}$. Meanwhile, mesoporous titania nanoparticles (MTNs) with a particle diameter of $373 \mathrm{~nm}$, a specific surface area of $236.3 \mathrm{~m}^{2} \mathrm{~g}^{-1}$, and a pore size of $2.8 \mathrm{~nm}$ were prepared by controlling the rate of hydrolysis. Magnetic colloidal crystals (a diameter of $10.2 \mu \mathrm{m}$ ) were formed by the aggregation of $\mathrm{Fe}_{3} \mathrm{O}_{4}$ and MTNs caused by the interface phenomena during solvent evaporation in emulsion. Even the anatase octahedrite produced from the colloidal crystal after a hydrothermal reaction retained a magnetic saturation of $2.8 \mathrm{emu} \mathrm{g}{ }^{-1}$. This study also investigates the photodegradation activity of our synthesized material as a photocatalyst, while utilizing its capability for magnetic separation to prove its usefulness in catalyst recycling
\end{abstract}

\section{Introduction}

The ability of a catalyst to reduce the activation energy of a chemical reaction and reaction time proves its vital application in various industrial processes. ${ }^{1}$ Moreover, catalysts are not depleted during a reaction, so methods to recycle and reuse catalysts are also popular research topics. ${ }^{2}$ General solid catalyst recycling methods include three main techniques: centrifugation, filtration, and magnetic separation. Centrifugation relies on the centrifugal force to separate the solid and liquid from a mixture, but is only appropriate for laboratory development rather than for industrial applications due to its time consuming procedures. ${ }^{3}$ Filtration relies on the varying particle sizes of a mixture so that only specific sized particles pass through the filter to establish separation between the catalyst and the solution. ${ }^{4}$

\footnotetext{
${ }^{a}$ Department of Chemical Engineering, National Taiwan University, No. 1, Sec. 4, Roosevelt Rd., Taipei 10617, Taiwan. E-mail: kevinwu@ntu.edu.tw

${ }^{b}$ Department of Chemistry, College of Science, King Saud University, Riyadh 11451, Saudi Arabia

${ }^{c}$ World Premier International (WPI) Research Centre for Materials Nanoarchitectonics (MANA), National Institute for Materials Science (NIMS), 1-1 Namiki, Tsukuba, Ibaraki 305-0044, Japan

${ }^{d}$ Division of Materials Science and Engineering, Faculty of Engineering, Hokkaido University, Kita 13 Nishi 8, Sapporo, Hokkaido, 060-8628, Japan.

E-mail: tetsu@eng.hokukai.ac.jp

$\dagger$ Electronic supplementary information (ESI) available. See DOI: 10.1039/ c5cp05005g

\# Equal contribution of the first two authors.
}

Yet filtration generally requires a vacuum source or high pressure to facilitate the process, which results in the additional cost and energy usage associated with filtration. Additionally, a long-term usage of the same filter causes particle deposition onto the filter pores, which reduces the efficiency of filtration. It is necessary to frequently replace the filter to prevent particle accumulation and maintain minimal filtration efficiency. ${ }^{5}$ Due to these difficulties presented by centrifugation and filtration methods, magnetic separation is the ideal technique for solid catalyst recycling. It is capable of cheaply and efficiently separating magnetic particles from a solution by applying an external magnetic field. ${ }^{6,7}$ The main prerequisite for using magnetic separation, however, is the catalyst's necessary magnetism.

Methods for the incorporation of magnetic particles within mesoporous catalysts are well studied, ${ }^{8}$ and mainly two general methods are utilized: the sol-gel method that creates a coreshell structure of magnetic cores and another material as a shell, ${ }^{9}$ and the direct growth of magnetic particles in the pores of another material. ${ }^{10}$ However, both methods have difficulties to overcome. For example, if the growth rate of titanium oxide for the core-shell structure was too slow, then the titanium oxide shell would be too thin to provide the necessary photocatalyst surface area. ${ }^{11,12}$ If the growth rates were too fast, then this would result in low particle dispersion and aggregation. Similarly, the method of direct magnetic particle growth in titanium oxide mesopores has a similar problem. ${ }^{13,14}$ In addition to the preliminary preparation of mesopores, the method 


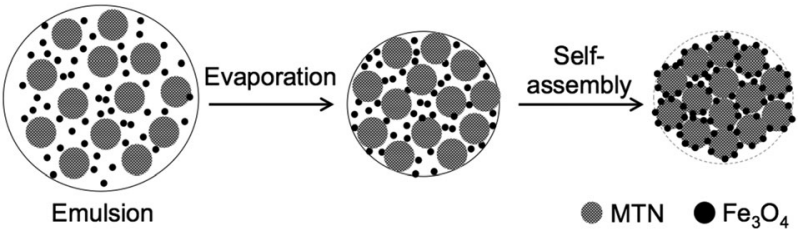

Fig. 1 Illustration of evaporation induced self-assembly (EISA) in emulsion for magnetic MTN colloidal crystals.

requires precise magnetic particle deposition control. ${ }^{15}$ Too few magnetic particles would result in the inadequate magnetic attraction during separation, while too many would result in magnetic particle deposition over the catalyst surface to cause low catalytic rates.

This study proposes a directed self-assembly process designed to incorporate magnetic particles within the titanium oxide sphere. We here choose to use mesoporous titania nanoparticles (MTNs) with a high surface area as photocatalysts and iron oxide $\left(\mathrm{Fe}_{3} \mathrm{O}_{4}\right)$ as the magnetic particle. As shown in Fig. 1, MTNs and $\mathrm{Fe}_{3} \mathrm{O}_{4}$ are suspended in the emulsion system in the form of droplets. The MTNs self-assemble into a colloidal crystal structured microparticle due to the interface phenomena during solvent evaporation. $\mathrm{Fe}_{3} \mathrm{O}_{4}$ is introduced into the colloidal crystal to result in the magnetized microparticle during the directed self-assembly of MTNs into the colloidal crystal structure. The resulting material consists of magnetic $\mathrm{Fe}_{3} \mathrm{O}_{4}$ and MTNs, and is abbreviated as M-MTNs hereafter. In addition to the structural and physical analysis of the M-MTNs, this study will investigate the activity of the M-MTNs as photocatalysts by measuring their organic degradation, while utilizing their capability for magnetic separation to prove their usefulness in catalyst recycling.

\section{Results and discussion}

Fig. 2 shows the XRD patterns of the different samples. The $\mathrm{Fe}_{3} \mathrm{O}_{4}$ patterns indicate the spinel crystalline, which is typical for iron oxide prepared by co-precipitation. After hydrothermal treatment, $\mathrm{Fe}_{3} \mathrm{O}_{4}$ is converted into $\alpha-\mathrm{Fe}_{2} \mathrm{O}_{3}$, which shows less apparent peaks than that of MTNs due to its lower starting

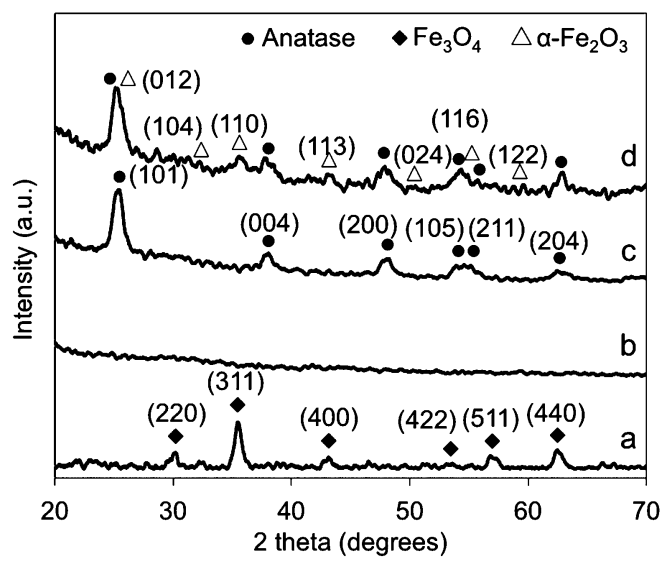

Fig. 2 XRD patterns: (a) $\mathrm{Fe}_{3} \mathrm{O}_{4}$, (b) MTNs, (c) MTN_HT, and (d) M-MTNs. amount during synthesis. The UV-Vis absorption of the samples was analyzed (data not shown). The adsorption wavelength for MTNs could be illustrated according to the following equation:

$$
E=\frac{h \lambda}{c}
$$

where $E$ is the band gap energy (eV), $h$ is Planck's constant $\left(6.6256 \times 10^{-34} \mathrm{~J} \mathrm{~s}\right), c$ is the speed of light $\left(2.9989 \times 10^{8} \mathrm{~m} \mathrm{~s}^{-1}\right)$, and $\lambda$ is the light's wavelength (nm). The MTNs after hydrothermal treatment without the addition of $\mathrm{Fe}_{3} \mathrm{O}_{4}$ (MTN_HT) exhibit characteristic peaks corresponding to anatase, which is an appropriate candidate as a photocatalyst. Since the $\mathrm{TiO}_{2}$ anatase has a band gap energy of $3.2 \mathrm{eV}$, it is possible to use eqn (1) to deduce that MTN_HT best absorbs and begins its photodegradation at a wavelength of $387.5 \mathrm{~nm}$. For the duration of this study, MTN_HT will serve as our standard photocatalyst to be compared with M-MTNs.

Fig. 3 shows the SEM image of all samples. Co-precipitated $\mathrm{Fe}_{3} \mathrm{O}_{4}$ mainly has a size distribution of $28.2 \mathrm{~nm}$, as shown in Fig. 3(a). The MTNs used here were prepared by a sol-gel method, using titanium tetraisopropoxide (TTIP) as a precursor for the hydrolysis-condensation reactions to yield $\mathrm{TiO}_{2}$. The overall reaction could be illustrated as:

$$
\mathrm{Ti}\left(\mathrm{OCH}\left(\mathrm{CH}_{3}\right)_{2}\right)_{4}+2 \mathrm{H}_{2} \mathrm{O} \rightarrow \mathrm{TiO}_{2}+4\left(\mathrm{CH}_{3}\right)_{2} \mathrm{CHOH}
$$

During the early stages of the reaction, the newly synthesized titanium oxide crystals would slowly and steadily form spheres of MTNs with a size of $373 \mathrm{~nm}$, as evaluated by SEM images in Fig. 3(b). The MTNs would then self-assemble to be M-MTN particles with a size of approximately $10.2 \mu \mathrm{m}$, as shown in Fig. 3(c and d). In the emulsion system, the solvent containing $\mathrm{Fe}_{3} \mathrm{O}_{4}$ and MTNs disperses in oil to form the emulsion drop. The evaporation of the solvent guides $\mathrm{Fe}_{3} \mathrm{O}_{4}$ and MTNs to align into the colloidal crystal. Since the particle
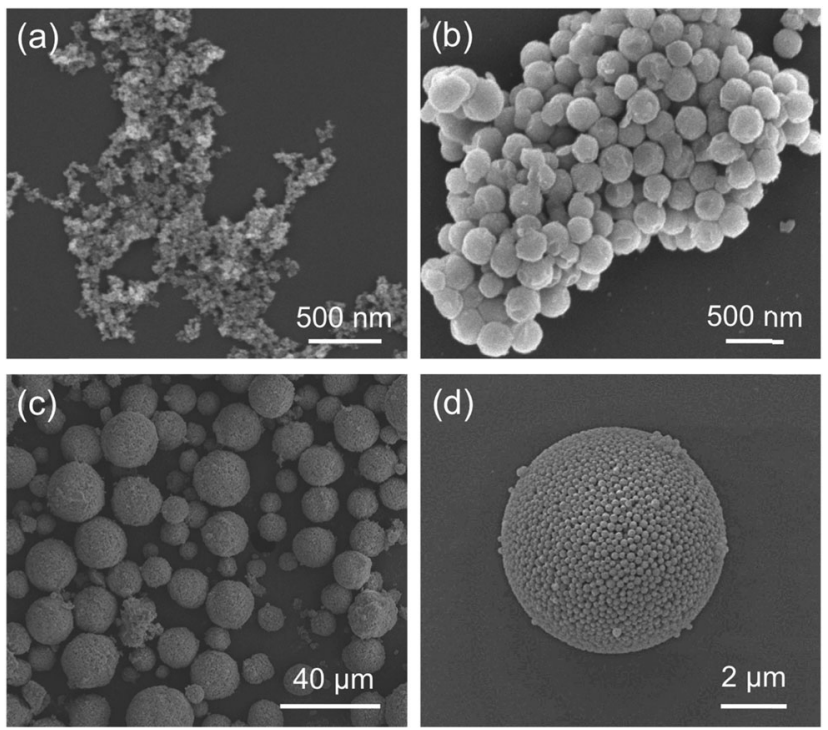

Fig. 3 SEM images: (a) $\mathrm{Fe}_{3} \mathrm{O}_{4}$, (b) MTNs, (c) and (d) M-MTN. 


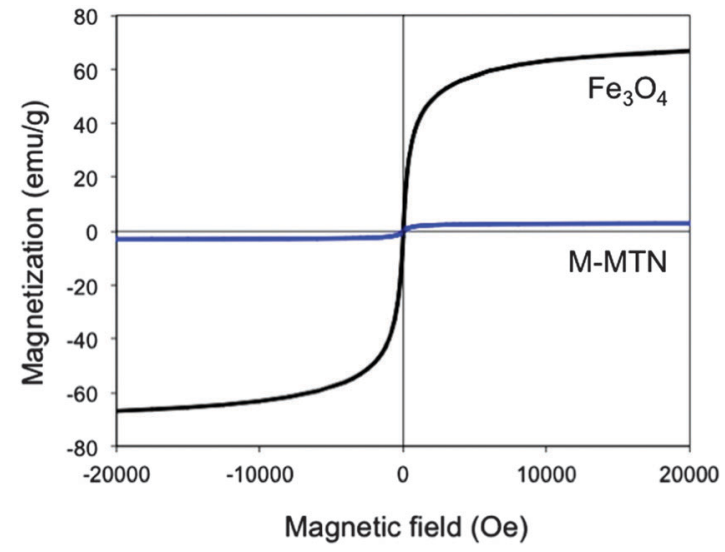

Fig. 4 Magnetic properties of $\mathrm{Fe}_{3} \mathrm{O}_{4}$ and M-MTNs.

size and the added amount of $\mathrm{Fe}_{3} \mathrm{O}_{4}$ are small compared to MTNs, the introduction of $\mathrm{Fe}_{3} \mathrm{O}_{4}$ does not hinder the formation of MTNs. The mesopores of MTNs are approximately 10-20 nm in size, which match the pore size distribution. As seen from Fig. 3, the cracks of the colloidal crystal are filled by $\mathrm{Fe}_{3} \mathrm{O}_{4}$ to give the microsphere magnetic properties (Fig. S1, ESI $\dagger$ ).

Fig. 4 shows that both the magnetic properties of $\mathrm{Fe}_{3} \mathrm{O}_{4}$ and M-MTNs have no corresponding hysteresis loops, which indicate that both are superparamagnetic. $\mathrm{Fe}_{3} \mathrm{O}_{4}$ has a magnetic saturation of $66.8 \mathrm{emu} \mathrm{g}^{-1}$, while M-MTNs have a magnetic saturation of $2.8 \mathrm{emu} \mathrm{g}^{-1}$. The magnetic saturation of M-MTNs is an order less than that of $\mathrm{Fe}_{3} \mathrm{O}_{4}$, because the mass ratio of $\mathrm{Fe}_{3} \mathrm{O}_{4}$ in MTNs is less than a tenth. Additionally, the hydrothermal reaction causes $\mathrm{Fe}_{3} \mathrm{O}_{4}$ to be converted into $\alpha-\mathrm{Fe}_{2} \mathrm{O}_{3}$, which further results in MTN's lower magnetic saturation.

Fig. 5 illustrates the nitrogen gas absorption and the pore size distribution of different samples, which are summarized in Table 1 along with the other characteristics. According to the IUPAC classification, MTNs prior to hydrothermal treatment are classified as type I as deduced by their hysteresis loop. This

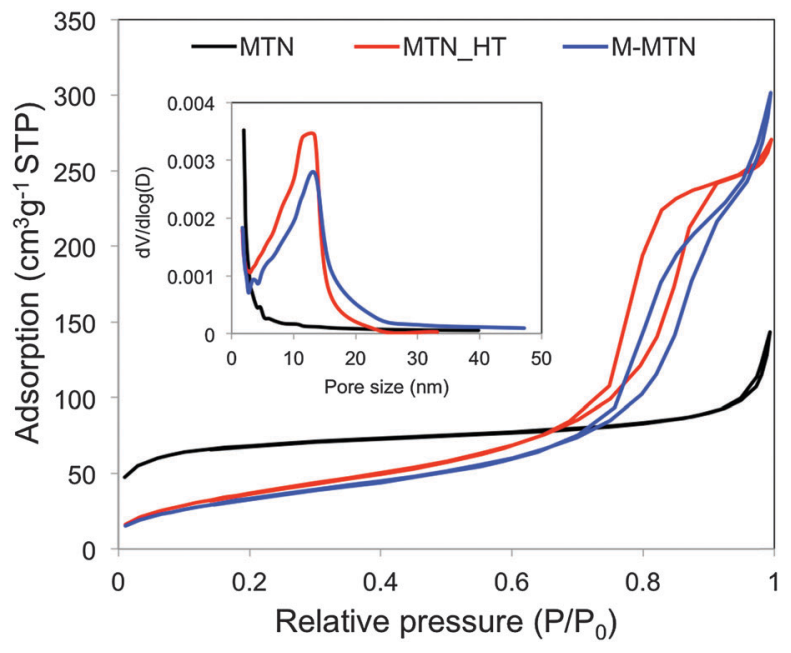

Fig. $5 \quad \mathrm{~N}_{2}$ adsorption-desorption isotherms and pore size distributions (inset).
Table 1 Summary of surface properties and photocatalytic activities of samples

\begin{tabular}{llllll}
\hline & $\begin{array}{l}\text { Surface } \\
\text { area }_{\left(\mathrm{m}^{2} \mathrm{~g}^{-1}\right)}\end{array}$ & $\begin{array}{l}\text { Pore } \\
\text { size } \\
(\mathrm{nm})\end{array}$ & Crystallinity & $\begin{array}{l}\text { Adsorption } \\
\text { ability } \\
\left(\mu \mathrm{g} \mathrm{mg} \mathrm{m}^{-1}\right)\end{array}$ & $\begin{array}{l}\text { Reaction } \\
\text { constant } \\
\left(k, \mathrm{~min}^{-1}\right)\end{array}$ \\
\hline MTN & 236.3 & 2.8 & Amorphous & N/A & N/A \\
MTN_HT & 142.7 & 10.9 & Anatase & 33.9 & 0.0448 \\
M-MTN & 129.4 & 12.3 & Anatase $+\alpha-\mathrm{Fe}_{2} \mathrm{O}_{3}$ & 18.5 & 0.0183 \\
\hline
\end{tabular}

indicates that gases are absorbed from the particle's micropores when under low air pressure. The pore size of MTNs was calculated to be $2.8 \mathrm{~nm}$ using Barrett-Joyner-Halenda (BJH) calculations. On the other hand, MTN_HT that has been obtained through hydrothermal treatment showed a type IV classification. This indicates that the majority of MTN_HT's pores are mesopores, which is supported by the BJH pore size calculations of $10.9 \mathrm{~nm}$. The difference in pore sizes may be due to the difference between the mass transfer coefficient of $\mathrm{TiO}_{2}$ and the vacancy during hydrothermal treatment. This difference results in the Kirkendall effect, which causes the aggregation of cracks to form mesopores and the conversion of $\mathrm{TiO}_{2}$ from amorphous to anatase. ${ }^{16}$ As the pore size increased, the material's specific surface area increased inversely from $236.3 \mathrm{~m}^{2} \mathrm{~g}^{-1}$ prior hydrothermal treatment to $142.7 \mathrm{~m}^{2} \mathrm{~g}^{-1}$ after hydrothermal treatment (Table 1).

The hysteresis loop of M-MTNs is similar to that of MTN_HT, where both are classified as type IV. ${ }^{17}$ As seen from the SEM images in Fig. 3(d), the cracks of the colloidal crystal particles are around $10-20 \mathrm{~nm}$. This indicates that the peak for pore size distribution of M-MTNs is more towards the right as compared to that of MTNs. M-MTNs exhibit an average pore diameter of $12.3 \mathrm{~nm}$ and a specific surface area of $129.4 \mathrm{~m}^{2} \mathrm{~g}^{-1}$. If the $\mathrm{Fe}_{3} \mathrm{O}_{4}$ contents were to be removed, then the specific surface area of M-MTNs would be $142.34 \mathrm{~m}^{2} \mathrm{~g}^{-1}$, which is similar to MTN_HT. Since the particle size of $\mathrm{Fe}_{3} \mathrm{O}_{4}(28.2 \mathrm{~nm})$ is an order larger than the diameter of MTN's mesopores $(2.8 \mathrm{~nm})$, particles of $\mathrm{Fe}_{3} \mathrm{O}_{4}$ would not occupy MTN's mesopores during self-assembly, which thus means that the photocatalyst retains its high specific surface area.

The general information on the photocatalyst ability to photodegrade Orange II is summarized in Fig. 6, where $C$ is the concentration of Orange II, $C^{*}$ is the starting concentration of Orange II, $C_{0}$ is the starting concentration of Orange II prior to $\mathrm{UV}$ radiation, and $t$ is the duration of $\mathrm{UV}$ radiation. Orange II degradation was not observed for the blank sample (without any addition of photocatalyst) under UV radiation. It is therefore deduced that the photocatalyst is the main factor for photodegradation upon its addition to the sample. As illustrated in Fig. 6(a), MTN_HT absorbed 44\% of Orange II while M-MTNs absorbed $29 \%$, which is $59 \%$ of what MTN_HT absorbed. It has been well known that particles are usually absorbed and aggregated around the pore opening during the absorption process for porous materials. For M-MTNs, the colloidal crystal structure of MTNs causes more closely packed particles, which result in fewer pores in contact with the solvent and thus lower absorption. Additionally, the possibility that 
(a)

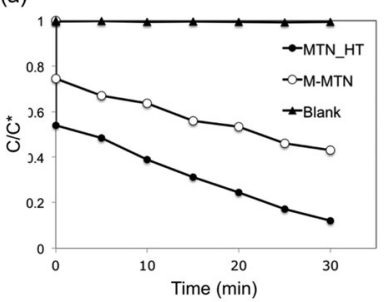

(c)

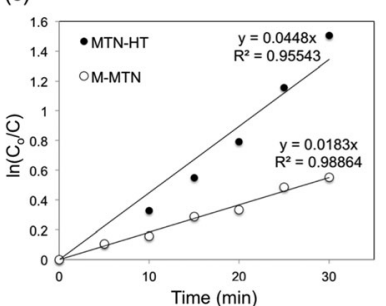

(b)

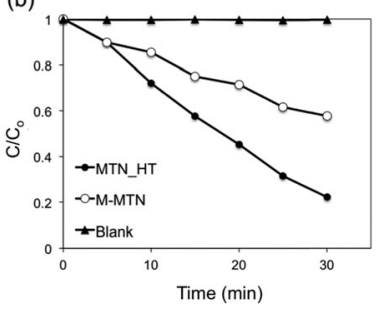

(d)

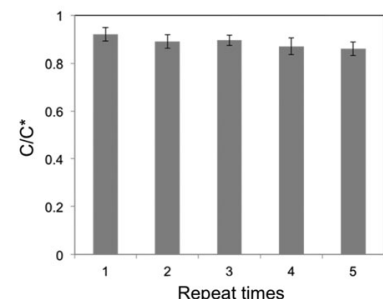

Fig. 6 Photocatalytic degradation of Orange II: (a) represented in initial concentration before adsorption, (b) represented in initial concentration before UV irradiation, (c) analyzed based on first-order kinetics. (d) Recycle experiment for Orange II removal.

$\mathrm{Fe}_{3} \mathrm{O}_{4}$ particles covered the openings of the pores could also be another reason for the lowered absorption. ${ }^{18}$

Fig. 6 illustrates the degradation kinetics after UV radiation. MTN_HT and M-MTNs both possess the ability for photodegradation, which could be described by the following first order equation:

$$
r=\frac{\mathrm{d} C}{\mathrm{~d} t}=k C
$$

where $r$ is the rate of Orange II degradation, $C$ is the concentration of Orange II, $k$ is a rate constant, and $t$ is the reaction time. Eqn (2) could be integrate to be:

$$
\ln \frac{C_{0}}{C}=k t
$$

where $C_{0}$ is the concentration of Orange II prior to UV radiation. Fig. 6(c) could be derived from Fig. 6(b) using the above equations to calculate the rate constant $(k)$. The $k$ value for M-MTNs was determined to be $0.0183 \mathrm{~min}^{-1}$, which is $40 \%$ of that for MTN_HT. Once again, this difference could be the result of $\alpha-\mathrm{Fe}_{2} \mathrm{O}_{3}$ covering the surface of MTNs, and thus resulting in blocked UV absorption and decreased photocatalytic degradation of M-MTNs.

Upon UV radiation for 90 minutes, M-MTNs were capable of removing large quantities of Orange II from the solvent $\left(C / C^{*}=\right.$ $90 \%$ ) through the processes of absorption and degradation. As shown in Fig. 7, M-MTNs could be efficiently separated from the solution with an external magnetic field given their magnetic properties from $\alpha-\mathrm{Fe}_{2} \mathrm{O}_{3}$. The effectiveness of recycled $\mathrm{M}$ MTNs was tested up to their fifth reuse, as shown in Fig. 6(d). Each sample received UV radiation for 90 minutes, and the recycled M-MTNs were still able to maintain an efficiency of approximately $90 \%$ with a standard deviation of $2.8 \%$.

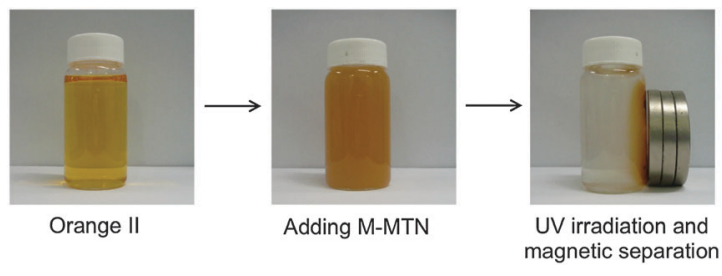

Fig. 7 Degradation of Orange II, and separation of M-MTNs before and after applying by magnetic field.

\section{Conclusions}

We were able to successfully synthesize closely packed colloidal crystal MTNs under a sol-gel system through self-assembly induced by the evaporation of the solvent. $\mathrm{Fe}_{3} \mathrm{O}_{4}$ particles were also introduced into the $\mathrm{TiO}_{2}$ sphere surface during synthesis to provide the microparticles with magnetic properties. Upon hydrothermal treatment, M-MTNs contained anatase crystals with a specific surface area of $129.4 \mathrm{~m}^{2} \mathrm{~g}^{-1}$ and a pore diameter of $12.3 \mathrm{~nm}$. M-MTNs were used to degrade Orange II for testing their photocatalytic activity and candidacy as recyclable photocatalysts. Even prior to the photocatalyst reaction, M-MTNs were capable of absorbing large quantities of Orange II due to their high specific surface area. M-MTNs then degraded Orange II upon UV radiation, with reaction rates of first-order equations. The magnetic recycling of M-MTNs was tested up to their fifth reuse with a consistent removal of approximately $90 \%$ of Orange II. This study illustrates the capability of the method of solvent evaporation to efficiently self-assemble multiple materials into a microparticle, while retaining the properties of each material as seen from our M-MTNs. We believe that our proposed synthesis method could be applied to a multitude of other materials with specific surface needs, and thus promoting the potential for materials science.

\section{Experimental section}

\section{Chemicals}

Ammonium hydroxide $\left(\mathrm{NH}_{4} \mathrm{OH}, 28 \%\right)$, hydrochloride acid $(\mathrm{HCl}, 37 \%)$, iron(III) chloride hexahydrate $\left(\mathrm{FeCl}_{3} \cdot 6 \mathrm{H}_{2} \mathrm{O}\right)$, Orange II, potassium chloride ( $\mathrm{KCl}$ ), and titanium(Iv) isopropoxide (abbreviated as TTIP, 97\%) were purchased from Sigma-Aldrich. Ferrous chloride, 4-hydrate $\left(\mathrm{FeCl}_{2} \cdot 4 \mathrm{H}_{2} \mathrm{O}\right)$ was purchased from J.T.Baker.

\section{Synthesis of mesoporous titania nanoparticles (MTNs)}

Mesoporous materials have been widely synthesized through various methods such as surfactant-templating processes and layer-by-layer processes. ${ }^{19-21}$ MTNs were obtained by the controlled hydrolysis of TTIP in an EtOH environment. ${ }^{22}$ In general, $2.2 \mathrm{~mL}$ of TTIP was added dropwise into $100 \mathrm{~mL}$ of pure EtOH containing $0.4 \mathrm{~mL}$ of $\mathrm{KCl}$ solution $(0.1 \mathrm{M})$. After stirring at ambient temperature for $60 \mathrm{~min}$, the white mixture was left stationary for 24 hours for aging. The precipitated product was collected by centrifugation, washed with EtOH, and dried under vacuum. 


\section{Synthesis of $\mathrm{Fe}_{3} \mathrm{O}_{4}$ nanoparticles}

$\mathrm{Fe}_{3} \mathrm{O}_{4}$ nanoparticles were prepared by co-precipitation method. ${ }^{23}$ $2 \mathrm{M} \mathrm{FeCl}_{2} \cdot 4 \mathrm{H}_{2} \mathrm{O}$ was prepared in $2 \mathrm{~mL}$ of $\mathrm{HCl}(2 \mathrm{M})$, and then added to $8 \mathrm{~mL}$ of $\mathrm{Fe}^{3+}$ aqueous solution $(1 \mathrm{M})$. This yellow mixture was mixed with $100 \mathrm{~mL}$ of $\mathrm{NH}_{4} \mathrm{OH}(0.7 \mathrm{M})$ to precipitate black $\mathrm{Fe}_{3} \mathrm{O}_{4}$. After stirring at ambient temperature for 30 minutes, $\mathrm{Fe}_{3} \mathrm{O}_{4}$ was collected by centrifuge and washed with DI water.

\section{Synthesis of $\mathrm{Fe}_{3} \mathrm{O}_{4} / \mathrm{MTN}$ colloidal crystals}

$0.4 \mathrm{~mL}$ of the aqueous solution containing $3 \mathrm{mg}$ of $\mathrm{Fe}_{3} \mathrm{O}_{4}$ and $30 \mathrm{mg}$ of the MTN was added into $20 \mathrm{~mL}$ of silica oil, stirred at $2000 \mathrm{rpm}$ for 10 minutes, and then left at $80{ }^{\circ} \mathrm{C}$ to evaporate the solvent. After 8 hours, the particles were washed with hexane, collected by centrifuge, and dried at ambient temperature. M-MTNs were obtained after a hydrothermal reaction at $180{ }^{\circ} \mathrm{C}$ for 12 hours.

\section{Characterization}

X-ray diffraction (XRD) patterns of samples were performed on a Rigaku ultima IV using $\mathrm{Cu} K \alpha$ radiation $(\lambda=0.154 \mathrm{~nm})$. The surface morphology and internal structure of products were observed using SEM (NovaTM Nano SEM) and TEM (JEOL JEM$1200 \mathrm{EX}$ II). The porous properties of samples were analyzed using N2 adsorption/desorption isotherms on a Micromeritics ASAP 2010 instrument, and all samples were degassed at $100{ }^{\circ} \mathrm{C}$ overnight before $\mathrm{N}_{2}$ adsorption. Surface area and pore size distribution were calculated using Brunauer-Emmett-Teller and Barrett-Joyner-Halenda methods, respectively. The magnetic properties of samples were studied using a SQUID (MPMS7 from Quantum Design Company of USA) magnetometer at a temperature of $300 \mathrm{~K}$.

\section{Photocatalysis}

$20 \mathrm{mg}$ of the catalyst and $20 \mathrm{~mL}$ of Orange II solution $\left(2 \times 10^{-5} \mathrm{M}\right)$ were stirred in the dark overnight to reach adsorption equilibrium. This aqueous slurry was then irradiated using a $4 \mathrm{~W} \mathrm{UV}$ lamp (wavelength at $254 \mathrm{~nm}$ ) and kept stirring in a dark environment. The concentration of Orange II was determined using a spectrophotometer (Jasco spectrophotometer V-670) at a wavelength of $484 \mathrm{~nm}$.

\section{Acknowledgements}

We would like to thank the Ministry of Science and Technology (MOST) for the funding support (103-2113-M-008-001; 104-2119M-008-010). S.M.A. and Y.Y. extends their sincere appreciation to the Deanship of Scientific Research at King Saud University for funding this Prolific Research Group (PRG-1436-19).

\section{References}

1 E. J. O'Neal and K. F. Jensen, ChemCatChem, 2014, 6, 3004.

2 H. Song, G. Li, X. Wang and Y. Chen, Microporous Mesoporous Mater., 2011, 139, 104.

3 K. Meixner, W. Fuchs, T. Valkova, K. Svardal, C. Loderer, M. Neureiter, G. Bochmann and B. Drosg, Sep. Purif. Technol., 2015, 145, 154.

4 N. Shamsuddin, D. B. Das and V. M. Starov, Chem. Eng. J., 2015, 276, 331.

5 A. Touffet, J. Baron, B. Welte, M. Joyeux, B. Teychene and H. Gallard, J. Membr. Sci., 2015, 489, 284.

6 W.-J. Liu, K. Tian, H. Jiang and H.-Q. Yu, Sci. Rep., 2013, 3, 2419.

7 D.-m. Lai, L. Deng, J. Li, B. Liao, Q.-X. Guo and Y. Fu, ChemSusChem, 2011, 4, 55.

8 Y.-C. Lee, C.-T. Chen, Y.-T. Chiu and K. C. W. Wu, ChemCatChem, 2013, 5, 2153.

9 Y. Zhu, X. Li, G. He and X. Qi, Chem. Commun., 2015, 51, 2991.

10 X.-Y. Wang, X.-P. Jiang, Y. Li, S. Zeng and Y.-W. Zhang, Int. J. Biol. Macromol., 2015, 75, 44.

11 S. Lee, K. Lee, W. D. Kim, S. Lee, D. J. Shin and D. C. Lee, J. Phys. Chem. C, 2014, 118, 23627.

12 H. Guo, D. Tian, L. Liu, Y. Wang, Y. Guo and X. Yang, J. Solid State Chem., 2013, 201, 137.

13 A. K. Patra, A. Dutta and A. Bhaumik, ACS Appl. Mater. Interfaces, 2012, 4, 5022.

14 L. Li, X. Li, H. Duan, X. Wang and C. Luo, Dalton Trans., 2014, 43, 8431.

15 S. Trey, R. T. Olsson, V. Strom, L. Berglund and M. Johansson, RSC Adv., 2014, 4, 35678.

16 H. Yin, Y. Wada, T. Kitamura, S. Kambe, S. Murasawa, H. Mori, T. Sakata and S. Yanagida, J. Mater. Chem., 2001, 11, 1694.

17 M. Marszewski and M. Jaroniec, RSC Adv., 2015, 5, 61960.

18 D. Losic, G. Triani, P. J. Evans, A. Atanacio, J. G. Mitchell and N. H. Voelcker, J. Mater. Chem., 2006, 16, 4029.

19 K. Ariga, Y. Yamauchi, G. Rydzek, Q. M. Ji, Y. Yonamine, K. C. W. Wu and J. P. Hill, Chem. Lett., 2014, 43, 36.

20 K. Ariga, A. Vinu, Y. Yamauchi, Q. Ji and J. P. Hill, Bull. Chem. Soc. Jpn., 2012, 85, 1.

21 V. Malgras, Q. Ji, Y. Kamachi, T. Mori, F.-K. Shieh, K. C.-W. Wu, K. Ariga and Y. Yamauchi, Bull. Chem. Soc. Jpn., 2015, 88, 1171.

22 K. C. W. Wu, Y. Yamauchi, C.-Y. Hong, Y.-H. Yang, Y.-H. Liang, T. Funatsu and M. Tsunoda, Chem. Commun., 2011, 47, 5232.

23 Y.-C. Lee, S. Dutta and K. C. W. Wu, ChemSusChem, 2014, $7,3241$. 